\title{
Weak Decay of Magnetized Pions
}

\author{
G. S. Bali, ${ }^{1,2}$ B. B. Brandt, ${ }^{3}$ G. Endrődi, ${ }^{3}$ and B. Gläßle ${ }^{4}$ \\ ${ }^{1}$ Institute for Theoretical Physics, Universität Regensburg, D-93040 Regensburg, Germany \\ ${ }^{2}$ Department of Theoretical Physics, Tata Institute of Fundamental Research, Homi Bhabha Road, Mumbai 400005, India \\ ${ }^{3}$ Institute for Theoretical Physics, Goethe Universität Frankfurt, D-60438 Frankfurt am Main, Germany \\ ${ }^{4}$ Zentrum für Datenverarbeitung (ZDV), Universität Tübingen, Wächterstr. 76, D-72074 Tübingen, Germany
}

(Received 17 June 2018; published 15 August 2018)

\begin{abstract}
The leptonic decay of charged pions is investigated in the presence of background magnetic fields. In this situation, Lorentz symmetry is broken, and new fundamental decay constants need to be introduced, associated with the decay via the vector part of the electroweak current. We calculate the magnetic field dependence of both the usual and a new decay constant nonperturbatively on the lattice. We employ both Wilson and staggered quarks and extrapolate the results to the continuum limit. With this nonperturbative input, we calculate the tree level electroweak amplitude for the full decay rate in strong magnetic fields. We find that the muonic decay of the charged pion is enhanced drastically by the magnetic field. We comment on possible astrophysical implications.
\end{abstract}

DOI: 10.1103/PhysRevLett.121.072001

Introduction.-Strong (electro)magnetic fields bear a significant impact on the physics of various systems, ranging from off-central heavy-ion collisions through the evolution of the early universe to magnetized neutron stars (magnetars). In particular, many novel phenomena emerge from the competition between electromagnetism and color interactions if the magnetic field $B$ becomes similar in magnitude to the strong interaction scale: $e B \sim \Lambda_{\mathrm{QCD}}^{2}$. If the time scale of the fluctuations in $B$ is larger than other relevant scales of the problem, it is reasonable to treat the magnetic field classically as a background field. For reviews on this subject, see for example Refs. [1,2].

Such a background magnetic field is known, for instance, to affect the phase diagram of quantum chromodynamics (QCD) [3-5]. For cold astrophysical environments, the lowemperature (hadronic) phase of QCD is particularly relevant. In this regime, a prime role is played by the lightest hadrons, i.e., pions and kaons. Specifically, their masses appear in the nuclear equation of state within compact stellar objects and, thus, influence their mass-radius relations. For stability and equilibrium analyses, the respective decay rates are equally important. Dominant cooling mechanisms for magnetars [6] involve (inverse) $\beta$ decay, photo-meson interactions, and pion decay [7]. Pions radiate energy via inverse Compton scattering until they decay, imprinting the spectrum of the subsequently

Published by the American Physical Society under the terms of the Creative Commons Attribution 4.0 International license. Further distribution of this work must maintain attribution to the author(s) and the published article's title, journal citation, and DOI. Funded by SCOAP ${ }^{3}$. produced neutrinos [8]. Strong electromagnetic fields are also created in violent astrophysical processes, such as neutron star mergers and supernova events, where weak nuclear reactions and decays govern cooling mechanisms and affect the neutrino spectrum [9].

The $B$ dependence of pion masses has been investigated in various settings, ranging from chiral perturbation theory $[10,11]$ through numerical lattice QCD simulations $[3,12-$ 14] to model approaches [15-20]. Less is known about the decay rates for nonzero magnetic fields. The decay constant for the neutral pion has been studied in chiral perturbation theory $[10,11,21]$ and in model settings $[15-19,22]$. The decay constant of the charged pion has only been discussed so far in chiral perturbation theory [11].

In this Letter, we investigate the magnetic field dependence of the decay rate of charged pions at zero temperature. We demonstrate that the previous studies in this direction are incomplete: in the presence of the magnetic field, both neutral and charged pions have two independent decay constants, of which only one has been investigated up to now. We determine both decay constants for charged pions nonperturbatively on the lattice, employing two different fermionic discretizations. Using this QCD input, we proceed to calculate the weak decay rate using leadingorder electroweak perturbation theory. For this calculation, we employ the lowest Landau level (LLL) approximation for the outgoing charged lepton state, which is a viable simplification for strong background magnetic fields. Our preliminary results using Wilson fermions on a reduced set of lattice spacings were presented in Ref. [23].

Pion decay constants.-The pion decay constant is related to the hadronic matrix elements $H_{\mu}$ of the weak interaction current between the vacuum and a pion state 
with momentum $p_{\mu}$. For $B=0$, parity dictates that the matrix element $\left\langle 0\left|\bar{d} \gamma_{\mu} u\right| \pi^{-}\right\rangle$vanishes, since the only Lorentz structure available is $p_{\mu}$ :

$$
\begin{aligned}
H_{\mu} & \equiv\left\langle 0\left|\bar{d}(x) \gamma_{\mu}\left(1-\gamma^{5}\right) u(x)\right| \pi^{-}(p)\right\rangle \\
& =-\left\langle 0\left|\bar{d}(x) \gamma_{\mu} \gamma^{5} u(x)\right| \pi^{-}(p)\right\rangle=-i e^{i p x} f_{\pi} p_{\mu} .
\end{aligned}
$$

The coefficient $f_{\pi}$ is the pion decay constant, which coincides for negatively and positively charged pions due to charge conjugation symmetry. Throughout this Letter we use the normalization where $f_{\pi} \approx 131 \mathrm{MeV}$ for a physical pion in the vacuum.

In the presence of a background electromagnetic field $F_{\mu \nu}$, the relation (1) takes a more general form. Exploiting Lorentz covariance, using the tensor $F_{\mu \nu}$ and the vector $p_{\mu}$, additional vector and axial vector combinations can be formed:

$$
\begin{aligned}
\left\langle 0\left|\bar{d}(x) \gamma_{\mu} \gamma^{5} u(x)\right| \pi^{-}(p)\right\rangle & =i e^{i p x}\left[f_{\pi} p_{\mu}+f_{\pi}^{\prime \prime} e F_{\mu \nu} p^{\nu}\right], \\
\left\langle 0\left|\bar{d}(x) \gamma_{\mu} u(x)\right| \pi^{-}(p)\right\rangle & =i e^{i p x}\left(i \frac{f_{\pi}^{\prime}}{2} \epsilon_{\mu \nu \rho \sigma} e F^{\nu \rho} p^{\sigma}\right),
\end{aligned}
$$

where $e$ denotes the elementary charge and we follow the convention $\epsilon^{0123}=+1$. Charge conjugation implies that the decay rate is the same for positively and negatively charged pions, and it is also independent of the direction of the magnetic field. This is ensured by the ratios $f_{\pi} / f_{\pi}^{\prime}$ and $f_{\pi} / f_{\pi}^{\prime \prime}$ being real, as we will see below. In our conventions, all three decay constants are real and positive. We remark that the new Lorentz structures also exist for matrix elements involving neutral pions.

We consider a background magnetic field $\mathbf{B}$ that points in the $z$ direction, implying $F_{21}=-F_{12}=B$. For a pion of mass $M_{\pi}$ with vanishing momentum along the magnetic field, $p_{3}=0$,

$$
H_{0}=-i e^{i M_{\pi} x_{0}} f_{\pi} M_{\pi}, \quad H_{3}=e^{i M_{\pi} x_{0}} f_{\pi}^{\prime} e B M_{\pi} .
$$

For charged states that are in the LLL, only these two components of $H_{\mu}$ contribute to the decay rate. (For details on this and further elements of the perturbative calculation, we refer to the Supplemental Material [24].) The decay constants $f_{\pi}$ and $f_{\pi}^{\prime}$ depend on the Lorentz scalars $F_{\mu \nu} F^{\mu \nu} / 2=B^{2}$ and $p_{\mu} p^{\mu}=M_{\pi}^{2}(B)$.

The matrix element of the vector current can also be interpreted from a different perspective: the magnetic field mixes the pion with the $\rho$ meson having zero spin projection along the magnetic field (i.e., $s_{3}=0$ ) [14]. Since the latter has the same quantum numbers as the $\mu=$ 3 component of the vector part of the electroweak current, this mixing gives rise to a nonzero value for the vector matrix element, the second relation of Eq. (2).

We mention that for nonzero temperature, an additional vector $u_{\mu}$ describing the thermal medium $\left(u_{0} \neq u_{i}\right)$ appears and leads to a splitting between spatial and temporal decay constants (see, for example, Ref. [22]). Here, we work at $T=0$, where this effect is absent. Furthermore, note that, the presence of the two terms $f_{\pi}$ and $f_{\pi}^{\prime \prime}$ in the first relation of Eq. (2) implies that the axial vector matrix element is different for indices $\mu=0,3$ and $\mu=1,2$, as was also found in Ref. [22]. However, for a purely magnetic background, the term involving $f_{\pi}^{\prime \prime}$ is absent from $H_{0}$ and $H_{3}$.

Pion decay rate.-The weak interaction matrix element (3) enters the rate of the leptonic decay process $\pi^{-}(p) \rightarrow \ell^{-}(k) \bar{\nu}_{\ell}(q)$, where $p, k$, and $q$ denote the four momenta of the pion, the charged lepton $\ell$ and the antineutrino $\bar{\nu}_{\ell}$, respectively. The decay into a muon $\ell=$ $\mu$ is the dominant channel, with a decay fraction of $99.98 \%$ [25] at $B=0$.

We work at the tree level of electroweak perturbation theory and employ the effective, four-fermion interaction with Fermi's constant $G$ as a coupling. Because of the currentcurrent structure of the effective electroweak Lagrangian $[26,27]$, the decay amplitude factorizes into leptonic and hadronic parts, $\mathcal{M}=G / \sqrt{2} \cos \theta_{c} L^{\mu} H_{\mu}$, where the Cabibbo angle $\theta_{c}$ entered, due to the mixing, between the down and strange quark mass eigenstates. The relevant hadronic components $H_{\mu}$ are shown in Eq. (3). Moreover, the leptonic component reads $L^{\mu} \equiv \bar{u}_{\ell}(k) \gamma^{\mu}\left(1-\gamma^{5}\right) v_{\nu}(q)$ in terms of the bispinor solutions $u_{\ell}$ and $v_{\nu}$.

The decay rate $\Gamma$ involves the modulus square of the amplitude, integrated over the phase space, and summed over the intrinsic quantum numbers of the outgoing asymptotic states. To find the latter for the charged lepton, we need the bispinor solutions of the Dirac equation for $B>0$. These are the so-called Landau levels - orbits localized in the spatial plane perpendicular to $\mathbf{B}$ with quantized radii. The Landau levels come with a multiplicity proportional to the flux $\Phi=$ $|e B| L^{2}$ of the magnetic field. In order to regulate this multiplicity, we need to assume that the outgoing states are defined in a finite spatial volume $V=L^{3}$. For the decay rate, such volume factors will cancel.

For strong fields, the dominant contribution stems from the lowest Landau level. The sum over the multiplicity of the LLL states gives [28]

$$
\sum_{\mathrm{LLL}} u_{\ell}(k) \bar{u}_{\ell}(k)=\frac{\Phi}{2 \pi}\left(\not \not_{\|}+m_{\ell}\right) \frac{1-\sigma^{12}}{2}
$$

where $\not_{\|}=k^{0} \gamma^{0}-k^{3} \gamma^{3}$ and $\sigma^{12}=i \gamma^{1} \gamma^{2}$ is the relativistic spin operator. Equation (4) reflects the fact that the LLL solutions have their spin antialigned with the magnetic field (since the lepton has negative charge) and are characterized only by the momentum along the $z$ direction (i.e., along $\mathbf{B}$ ). Because of angular momentum conservation, the antineutrino spin is also aligned with the magnetic field. Moreover, the right-handedness of the antineutrino also sets the 
direction of its momentum to be parallel to the magnetic field.

Having determined $|\mathcal{M}|^{2}$, we finally need to integrate over the phase space for the outgoing particles. The resulting decay rate reads

$$
\Gamma(B)=|e B| \frac{G^{2}}{4 \pi} \cos ^{2} \theta_{c}\left|f_{\pi}+i f_{\pi}^{\prime} e B\right|^{2} \frac{m_{\ell}^{2}}{M_{\pi}} .
$$

As anticipated above, the decay rate only depends on the magnitude of $B$, due to the absence of an interference term in $\left|f_{\pi}+i f_{\pi}^{\prime} e B\right|^{2}=f_{\pi}^{2}+\left[f_{\pi}^{\prime} e B\right]^{2}$. Dividing by the $B=0$ result [26], the dependence on $G$ and $\theta_{c}$ cancels:

$\frac{\Gamma(B)}{\Gamma(0)}=\frac{f_{\pi}^{2}+\left[f_{\pi}^{\prime} e B\right]^{2}}{f_{\pi}^{2}(0)}\left[1-\frac{m_{\ell}^{2}}{M_{\pi}^{2}(0)}\right]^{-2} \frac{2|e B|}{M_{\pi}(0) M_{\pi}(B)}$.

We stress that this result was obtained using the LLL approximation, which is in general valid for strong fields $[2,29]$. For the leading-order perturbative decay rate, higher Landau levels turn out to give zero contribution for $e B>M_{\pi}^{2}(0)-m_{\ell}^{2}$.

Lattice setup.-Equation (6) contains three nonperturbative parameters that describe the response of the pion to the background field: $M_{\pi}(B), f_{\pi}(B)$, and $f_{\pi}^{\prime}(B)$. We calculate these via two independent sets of lattice QCD simulations. First, we work with quenched Wilson quarks. The zerotemperature ensembles, generated and analyzed in Ref. [14], are supplemented by a fourth, finer lattice ensemble, so that the lattice spacing spans $0.047 \mathrm{fm} \leq a \leq 0.124 \mathrm{fm}$. The $B=0$ pion mass is set to $M_{\pi}(0) \approx 415 \mathrm{MeV}$. To remove $B$-dependent $\mathcal{O}\left(a^{2}\right)$ effects on quark masses, the bare mass parameters are tuned to fall on the magnetic field dependent line of constant physics determined in Ref. [14].

In the second set of simulations, we work with $N_{f}=$ $2+1$ flavors of dynamical staggered fermions, using the ensembles of Refs. [3,30]. The employed lattice spacings lie in the range $0.1 \mathrm{fm} \leq a \leq 0.22 \mathrm{fm}$, and the quark masses are set to their physical values [31] such that $M_{\pi}(0) \approx 135 \mathrm{MeV}$. For both formulations, we perform a continuum extrapolation based on the available four lattice spacings. This enables us to quantify the systematics related to the differences between the two approaches: heavier-than-physical versus physical pion mass and quenched versus dynamical quarks. We remark that simulations with dynamical Wilson quarks in the presence of a background magnetic field would require computational resources that are by orders of magnitude larger than those used for the current study.

The general measurement strategy involves the analysis of the matrix elements $H_{0}$ and $H_{3}$ of Eq. (3). These are encoded in the spatially averaged Euclidean correlators $C_{\mathcal{O P}}(t)=\left\langle\sum_{\mathbf{x}} \mathcal{O}(\mathbf{x}, t) P^{\dagger}(\mathbf{0}, 0)\right\rangle$ with $\mathcal{O}$ being either of $P=\bar{u} \gamma^{5} d, A=\bar{u} \gamma_{0} \gamma^{5} d$ or $V=\bar{u} \gamma_{3} d$. In the large- $t$ limit, the dominant contribution to the spectral representation of all three correlators comes from a pion state. We fit the three correlators using

$$
C_{\mathcal{O P}}(t)=c_{\mathcal{O P}}\left[e^{-M_{\pi} t} \pm e^{-M_{\pi}\left(N_{t}-t\right)}\right]
$$

where the positive sign is taken for $C_{P P}$ and the negative for $C_{A P}$ and $C_{V P}$ due to the time reversal properties of the correlators. The decay constants are extracted via

$$
f_{\pi}=Z_{A} \frac{\sqrt{2} c_{A P}}{\sqrt{M_{\pi} c_{P P}}}, \quad i f_{\pi}^{\prime} e B=Z_{V} \frac{\sqrt{2} c_{V P}}{\sqrt{M_{\pi} c_{P P}}},
$$

where $Z_{A}$ and $Z_{V}$ are the multiplicative renormalization constants of the axial vector and vector currents.

For Wilson quarks, we employ smeared pseudoscalar sources (for more details, see Ref. [14]) and fit all three correlators simultaneously. For the staggered analysis, we work with point sources and fit the $C_{A P}$ and $C_{P P}$ correlators to find $M_{\pi}$ and $f_{\pi}$. In a second step, volume sources are employed for $C_{V P} / C_{A P}$ to enhance the signal in $f_{\pi}^{\prime} / f_{\pi}$. The staggered discretization of $A$ and $V$ requires operators nonlocal in Euclidean time and has been worked out in Ref. [32]. For staggered quarks and the currents we use the renormalization constants are trivial, $Z_{A}=Z_{V}=1$. For Wilson quarks, this is not the case; nevertheless, these ultraviolet quantities are expected to be independent of the magnetic field. We employ the $B=0$ nonperturbative results of Ref. [33] (see also Ref. [34]) and fit these in combination with the asymptotic perturbative twoloop results of Ref. [35] (see also Ref. [36]) to a Padé parametrization.

Results.-Inspecting the $B>0$ correlation functions, we see clear signals for $C_{V P}$ (see the Supplemental Material [24]), which vanishes at $B=0$. The mass and the decay constants are extracted using the fits described in Eqs. (7) and (8). For the complete magnetic field range, the pion mass is found to be described within $5 \%$ by the formula

$$
M_{\pi} / M_{\pi}(0)=\sqrt{1+|e B| / M_{\pi}^{2}(0)}
$$

which assumes pions to be pointlike free scalars. This has been observed many times in the literature, both using dynamical staggered [3], quenched Wilson [12,14], and quenched overlap quarks [13].

The normalized combinations $f_{\pi} / f_{\pi}(0)$ and $f_{\pi}^{\prime} e B / f_{\pi}(0)$ are shown for four lattice spacings in Fig. 1, both for staggered and for Wilson fermions. To parametrize the $B$ dependence, we found that it is advantageous to consider polynomial fits for the amplitudes $f_{\pi} M_{\pi}$ and $f_{\pi}^{\prime} e B M_{\pi}$ of the matrix elements of Eq. (3). The continuum extrapolation is carried out by including lattice artefacts of $\mathcal{O}(a)$ (for Wilson) and $\mathcal{O}\left(a^{2}\right)$ (for staggered) in the coefficients. Specifically, the parametrizations of the individual decay constants read 

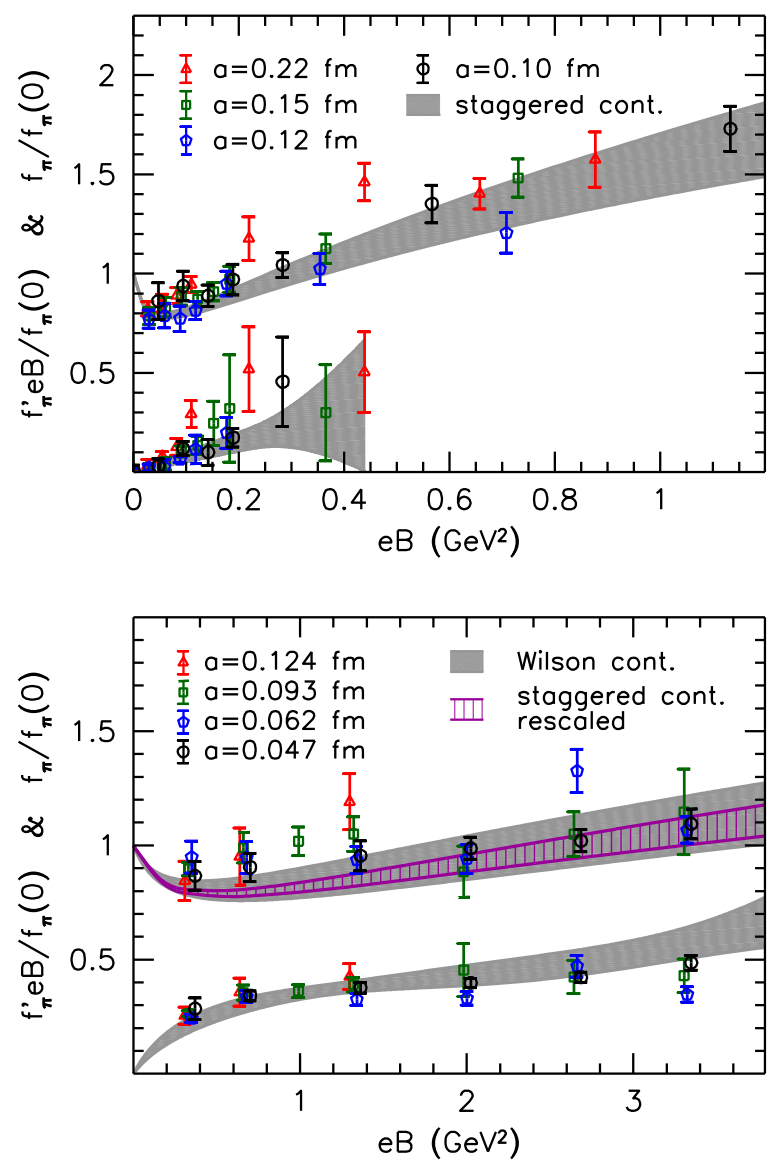

FIG. 1. Continuum extrapolation (gray bands) of the decay constants for staggered (upper panel) and Wilson quarks (lower panel). Both panels include results for $f_{\pi} / f_{\pi}(0)$ (upper points) and for $f_{\pi}^{\prime} e B / f_{\pi}(0)$ (lower points). The staggered results were obtained at the physical point, while the Wilson results correspond to a $B=0$ pion mass of $M_{\pi}(0)=415 \mathrm{MeV}$. For $f_{\pi} / f_{\pi}(0)$ we also compare the two continuum extrapolations after a rescaling of the magnetic field for the staggered curve (purple band; see the text for details).

$$
\begin{aligned}
& f_{\pi} / f_{\pi}(0)=\left[1+c_{1}|e B|\right] M_{\pi}(0) / M_{\pi}, \\
& f_{\pi}^{\prime} / f_{\pi}(0)=\left[d_{0}+d_{1}|e B|+d_{2}|e B|^{2}\right] M_{\pi}(0) / M_{\pi},
\end{aligned}
$$

and $M_{\pi} / M_{\pi}(0)$ is taken from Eq. (9). The quality of the staggered data for $f_{\pi}^{\prime} / f_{\pi}(0)$ only allows for a fit with $d_{1}=d_{2}=0$. For larger magnetic fields, we also include a systematic error estimated using the uncertainties of the data at high $B$. Ideally, the analysis in this region should be complemented by additional finer ensembles to make the continuum extrapolation of $f_{\pi}^{\prime}$ more robust. Within our range of $B$ fields, however, the decay rate and its uncertainty are dominated by $f_{\pi}$.

Motivated by the dependence of $M_{\pi} / M_{\pi}(0)$ on the scaling variable $e B / M_{\pi}^{2}(0)$, we compare the continuum extrapolated Wilson results (obtained for $M_{\pi}(0)=415 \mathrm{MeV}$ ) to the staggered data (obtained for physical pion masses), after rescaling the magnetic field for the latter. In particular, we take the staggered results for $f_{\pi} / f_{\pi}(0)$ at the magnetic field $e B(415 / 135)^{2}$. The resulting curve is also included in the lower panel of Fig. 1, revealing a nice agreement between the two approaches. In particular, the slope at the origin is found to be $-16.9(3) \mathrm{GeV}^{-2}$ for staggered and $-1.7(2) \mathrm{GeV}^{-2}$ for Wilson-the ratio of which is consistent with the squared pion mass ratio.

For low magnetic fields, the ratio $f_{\pi}^{\prime} / f_{\pi}(0)$ approaches a constant so that in this case the two discretizations can be compared to each other without a similar rescaling. We indeed find consistent results: $f_{\pi}^{\prime} / f_{\pi}(0)=0.8(2) \mathrm{GeV}^{-2}$ for staggered and $1.2(3) \mathrm{GeV}^{-2}$ for Wilson, respectively. We note that, the errors of the staggered data for this decay constant increase quickly as $B$ grows, rendering a comparison for higher magnetic fields inconclusive. We mention moreover that due to the different treatment of sea quark loops in the two approaches, the observed agreement is rather surprising and calls for a better understanding of the role of dynamical quarks in the Wilson setup.

To determine the decay rate (6), we employ the continuum extrapolated staggered results. On the basis of the above comparisons, we also consider the Wilson results, using a rescaling to the physical point as explained above. For the pion mass, we use the analytic dependence (9), including a 5\% systematic error. The so obtained curves for the muonic decay rate are shown in Fig. 2 for magnetic fields $e B \leq 0.45 \mathrm{GeV}^{2}$, where both staggered and (rescaled) Wilson results are available. The decay rate is enhanced drastically by the magnetic field: for $e B \approx$ $0.3 \mathrm{GeV}^{2}$ we observe an almost 50-fold increase with respect to $B=0$. We note that, while the ordinary decay mechanism dominates in our study, the contribution of the

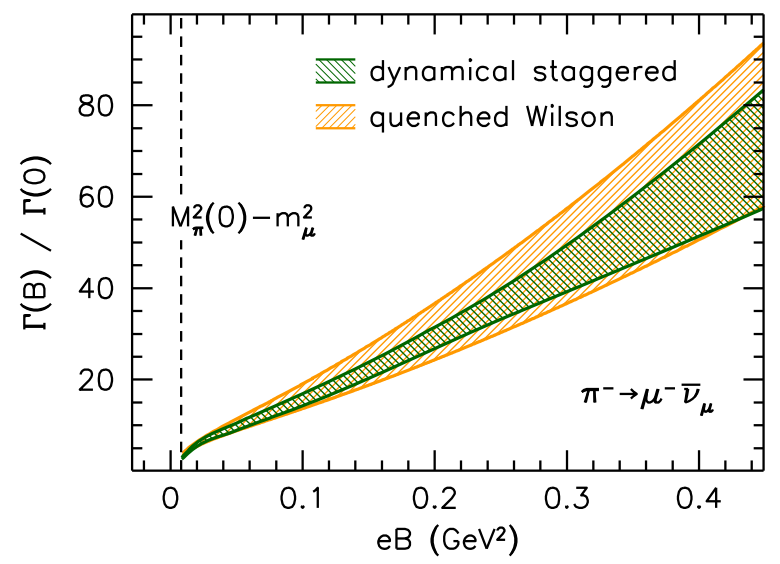

FIG. 2. The muonic decay rate in units of its $B=0$ value using the continuum extrapolated staggered results with physical quark masses (green). For comparison, the continuum extrapolated Wilson data at higher-than-physical quark masses are also included after a rescaling of the magnetic field by the squared pion mass (yellow). The LLL approximation we employed for the decay rate is valid for $e B>M_{\pi}^{2}(0)-m_{\mu}^{2}$, marked by the dashed vertical line. 
new vector decay constant $f_{\pi}^{\prime}$ grows to about $10 \%$ of the total decay rate at the largest magnetic field of Fig. 2.

We remark that Eq. (6), supplemented by our staggered lattice results, suggests the decay rate $K^{-} \rightarrow \mu^{-} \bar{\nu}_{\mu}$ to be enhanced only by a factor of about two at $e B \approx 0.3 \mathrm{GeV}^{2}$. This is mainly due to the larger mass of the kaon. Finally, the pion decay rate into electrons undergoes an enhancement by a factor of about ten. In fact, according to Eq. (5) the ratio of muonic and electronic decay rates becomes independent of the magnetic field,

$\Gamma(B)_{\pi \rightarrow e \bar{\nu}_{e}} / \Gamma(B)_{\pi \rightarrow \mu \bar{\nu}_{\mu}}=\left(m_{e} / m_{\mu}\right)^{2} \approx 2.27 \times 10^{-5}$,

and is by about a factor of 5.4 smaller than the corresponding fraction $1.23 \times 10^{-4}$ at $B=0$.

Conclusions. - In this Letter, we computed the rate for the leptonic decay of charged pions in the presence of strong background magnetic fields. The result is given by Eq. (6), for which we employed electroweak perturbation theory and the lowest-Landau-level approximation for the outgoing charged lepton $\ell$, valid for strong fields. Including higher-order terms in the electroweak calculation (see, e.g., Refs. [37,38]), as well as going beyond the lowest Landau level is possible, allowing one to systematically improve this result. In this case also, the constant $f_{\pi}^{\prime \prime}$, that we have not determined here, may enter.

We demonstrated that - besides the ordinary pion decay constant $f_{\pi}$ - the decay rate depends on an additional fundamental parameter $f_{\pi}^{\prime}$. The latter decay constant characterizes a new decay mechanism that becomes available for nonzero magnetic fields. We calculated both decay constants, together with the pion mass, using lattice simulations employing dynamical staggered quarks with physical masses, and also compared to the results of quenched Wilson simulations with heavier-than-physical quarks. For both cases, continuum extrapolations were carried out to eliminate lattice discretization errors. For low magnetic fields, we obtain for the new decay constant $f_{\pi}^{\prime}=0.10(2) \mathrm{GeV}^{-1}+\mathcal{O}(B)$.

Our final result for the full decay rate is visualized in Fig. 2, revealing a dramatic enhancement of the rate or, correspondingly, a drastic reduction of the mean lifetime $\tau_{\pi}=1 / \Gamma$. A typical $B>0$ lifetime is

$$
\tau_{\pi}=5 \times 10^{-10} \mathrm{~s} \text { for } B \approx 0.3 \mathrm{GeV}^{2} / e \approx 5 \times 10^{15} \mathrm{~T} .
$$

Since lifetimes of magnetic fields in off-central heavy-ion collisions are by 14-15 orders of magnitude smaller [1], it is clear that this effect will not result in any observable predictions for heavy-ion phenomenology. However, the $B$ dependence of weak decays is expected to be essential in astrophysical environments. (Notice that, the upper limit for magnetic field strengths in the core of magnetized neutron stars is thought to be around $B=10^{14}-10^{16} \mathrm{~T}$ $[39,40]$.) Indeed, for $B=0$, the pion mean lifetime and the time scale for cooling via inverse Compton scattering are roughly comparable [8]. Thus, a reduction in $\tau_{\pi}$ will inevitably decrease radiation energy loss of pions and result in a harder neutrino spectrum.

Similarly to the pion decay rate, the magnetic field will have an impact on (inverse) $\beta$-decay rates and nucleon electroweak transition form factors. Indeed, an enhancement by the magnetic field is expected for processes involving nucleons as well [41-43], see, e.g., the review [44]. The tools developed in the present Letter will also be useful to study these effects that are relevant for cold and magnetized environments.

This research was funded by the DFG (Emmy Noether Programme EN 1064/2-1 and SFB/TRR 55). G. B. thanks Basudeb Dasgupta for discussion. The computations were carried out on the GPU, iDataCool and Athene 2 clusters of Universität Regensburg.

[1] D. Kharzeev, K. Landsteiner, A. Schmitt, and H.-U. Yee, Strongly interacting matter in magnetic fields, Lect. Notes Phys. 871, 1 (2013).

[2] V. A. Miransky and I. A. Shovkovy, Quantum field theory in a magnetic field: From quantum chromodynamics to graphene and Dirac semimetals, Phys. Rep. 576, 1 (2015).

[3] G. Bali, F. Bruckmann, G. Endrődi, Z. Fodor, S. Katz, S. Krieg, A. Schäfer, and K. K. Szabó, The QCD phase diagram for external magnetic fields, J. High Energy Phys. 02 (2012) 044.

[4] G. Endrôdi, Critical point in the QCD phase diagram for extremely strong background magnetic fields, J. High Energy Phys. 07 (2015) 173.

[5] J. O. Andersen, W. R. Naylor, and A. Tranberg, Phase diagram of QCD in a magnetic field: A review, Rev. Mod. Phys. 88, 025001 (2016).

[6] R. C. Duncan and C. Thompson, Formation of very strongly magnetized neutron stars-implications for gamma-ray bursts, Astrophys. J. 392, L9 (1992).

[7] E. Waxman and J. N. Bahcall, High-Energy Neutrinos from Cosmological Gamma-Ray Burst Fireballs, Phys. Rev. Lett. 78, 2292 (1997).

[8] B. Zhang, Z. G. Dai, and P. Mészáros, High-energy neutrinos from magnetars, Astrophys. J. 595, 346 (2003).

[9] B. D. Metzger, T. A. Thompson, and E. Quataert, A magnetar origin for the kilonova ejecta in GW170817, Astrophys. J. 856, 101 (2018).

[10] N. O. Agasian and I. Shushpanov, Gell-Mann-Oakes-Renner relation in a magnetic field at finite temperature, J. High Energy Phys. 10 (2001) 006.

[11] J. O. Andersen, Chiral perturbation theory in a magnetic background-finite-temperature effects, J. High Energy Phys. 10 (2012) 005.

[12] Y. Hidaka and A. Yamamoto, Charged vector mesons in a strong magnetic field, Phys. Rev. D 87, 094502 (2013).

[13] E. V. Luschevskaya, O. E. Solovjeva, O. A. Kochetkov, and O. V. Teryaev, Magnetic polarizabilities of light mesons in SU(3) lattice gauge theory, Nucl. Phys. B898, 627 (2015). 
[14] G. S. Bali, B. B. Brandt, G. Endrődi, and B. Gläßle, Meson masses in electromagnetic fields with Wilson fermions, Phys. Rev. D 97, 034505 (2018).

[15] S. Fayazbakhsh, S. Sadeghian, and N. Sadooghi, Properties of neutral mesons in a hot and magnetized quark matter, Phys. Rev. D 86, 085042 (2012).

[16] S. S. Avancini, R. L. S. Farias, M. Benghi Pinto, W. R. Tavares, and V.S. Timóteo, $\pi_{0}$ pole mass calculation in a strong magnetic field and lattice constraints, Phys. Lett. B 767, 247 (2017).

[17] R. Zhang, W.-J. Fu, and Y.-X. Liu, Properties of mesons in a strong magnetic field, Eur. Phys. J. C 76, 307 (2016).

[18] S. Mao and Y. Wang, Effect of quark dimension reduction on Goldstone mode in magnetic field, Phys. Rev. D 96, 034004 (2017).

[19] D. Gómez Dumm, M. Izzo Villafañe, and N. N. Scoccola, Neutral meson properties under an external magnetic field in nonlocal chiral quark models, Phys. Rev. D 97, 034025 (2018).

[20] M. Coppola, D. Gómez Dumm, and N. N. Scoccola, Charged pion masses under strong magnetic fields in the NJL model, Phys. Lett. B 782, 155 (2018).

[21] J. O. Andersen, Thermal pions in a magnetic background, Phys. Rev. D 86, 025020 (2012).

[22] S. Fayazbakhsh and N. Sadooghi, Weak decay constant of neutral pions in a hot and magnetized quark matter, Phys. Rev. D 88, 065030 (2013).

[23] G. S. Bali, B. B. Brandt, G. Endrődi, and B. Gläßle, Pion decay in magnetic fields, EPJ Web Conf. 175, 13005 (2018).

[24] See Supplemental Material at http://link.aps.org/ supplemental/10.1103/PhysRevLett.121.072001 for details of the perturbative calculation and an example of the lattice correlators for strong magnetic fields.

[25] K. A. Olive et al. (Particle Data Group Collaboration), Review of particle physics, Chin. Phys. C 38, 090001 (2014).

[26] L. Okun, Leptons and Quarks (Elsevier Science, New York, 2013).

[27] M. Schwartz, Quantum Field Theory and the Standard Model (Cambridge University Press, Cambridge, England, 2014).

[28] K. Bhattacharya, Solution of the Dirac equation in presence of an uniform magnetic field, Phys. Rev. D 96, 074506 (2017).

[29] F. Bruckmann, G. Endrődi, M. Giordano, S. D. Katz, T. G. Kovács, F. Pittler, and J. Wellnhofer, Landau levels in QCD, Phys. Rev. D 96, 074506 (2017).
[30] G. S. Bali, F. Bruckmann, G. Endrődi, Z. Fodor, S. D. Katz, and A. Schäfer, QCD quark condensate in external magnetic fields, Phys. Rev. D 86, 071502 (2012).

[31] S. Borsányi, G. Endrődi, Z. Fodor, A. Jakovác, S. D. Katz, S. Krieg, C. Ratti, and K. K. Szabó, The QCD equation of state with dynamical quarks, J. High Energy Phys. 11 (2010) 077.

[32] G. W. Kilcup and S. R. Sharpe, A tool kit for staggered fermions, Nucl. Phys. B283, 493 (1987).

[33] V. Gimenez, L. Giusti, F. Rapuano, and M. Talevi, Nonperturbative renormalization of quark bilinears, Nucl. Phys. B531, 429 (1998).

[34] M. Göckeler, R. Horsley, H. Oelrich, H. Perlt, D. Petters, P. E. L. Rakow, A. Schäfer, G. Schierholz, and A. Schiller, Nonperturbative renormalization of composite operators in lattice QCD, Nucl. Phys. B544, 699 (1999).

[35] A. Skouroupathis and H. Panagopoulos, Two-loop renormalization of vector, axial-vector and tensor fermion bilinears on the lattice, Phys. Rev. D 79, 094508 (2009).

[36] G. S. Bali, F. Bursa, L. Castagnini, S. Collins, L. Del Debbio, B. Lucini, and M. Panero, Mesons in large-N QCD, J. High Energy Phys. 06 (2013) 071.

[37] V. Lubicz, G. Martinelli, C. T. Sachrajda, F. Sanfilippo, S. Simula, and N. Tantalo, Finite-volume QED corrections to decay amplitudes in lattice QCD, Phys. Rev. D 95, 034504 (2017).

[38] A. Patella, QED corrections to hadronic observables, Proc. Sci., LATTICE2016 (2017) 020.

[39] D. Lai and S. L. Shapiro, Cold equation of state in a strong magnetic field-Effects of inverse beta-decay, Astrophys. J. 383, 745 (1991).

[40] E. J. Ferrer, V. de la Incera, J. P. Keith, I. Portillo, and P. L. Springsteen, Equation of state of a dense and magnetized fermion system, Phys. Rev. C 82, 065802 (2010).

[41] J. J. Matese and R. F. O'Connell, Neutron beta decay in a uniform constant magnetic field, Phys. Rev. 180, 1289 (1969).

[42] L. Fassio-Canuto, Neutron beta decay in a strong magnetic field, Phys. Rev. 187, 2141 (1969).

[43] S. Shinkevich and A. Studenikin, Relativistic theory of inverse beta decay of polarized neutron in strong magnetic field, Pramana J. Phys. 65, 215 (2005).

[44] C. Giunti and A. Studenikin, Neutrino electromagnetic interactions: A window to new physics, Rev. Mod. Phys. 87, 531 (2015). 